\title{
Repeated loss of variation in insect ovary morphology highlights the role of developmental constraint in life-history evolution
}

\author{
Samuel H. Church ${ }^{*}$, Bruno A. S. de Medeiros ${ }^{1,2}$, Seth Donoughe ${ }^{1,3}$, Nicole L. Márquez Reyes ${ }^{4}$, Cassandra \\ G. Extavour ${ }^{1,5 *}$ \\ ${ }^{1}$ Department of Organismic and Evolutionary Biology, Harvard University, Cambridge, MA 02138, USA \\ 2 Smithsonian Tropical Research Institute, Panama City, Panama \\ ${ }^{3}$ Department of Molecular Genetics and Cell Biology, University of Chicago, Chicago, IL 60637, USA \\ ${ }^{4}$ Department of Biology, Universidad de Puerto Rico en Cayey, Cayey 00736, PR \\ ${ }^{5}$ Department of Molecular \& Cellular Biology, Harvard University, Cambridge, MA 02138, USA \\ * Corresponding author
}

\begin{abstract}
The number of offspring an organism can produce is a key component of its evolutionary fitness and lifehistory. Here we perform a test of the hypothesized trade off between the number and size of offspring using thousands of descriptions of the number of egg-producing compartments in the insect ovary (ovarioles), a common proxy for potential offspring number in insects. We find evidence of a negative relationship between egg size and ovariole number when accounting for adult body size. However in contrast to prior claims, we note that this relationship is not generalizable across all insect clades, and we highlight several factors that may have contributed to this size-number trade off being stated as a general rule in previous studies. We reconstruct the evolutionary history of the nurse cell arrangement within the ovariole, and show that the diversification of ovariole number and egg size have both been largely independent of nurse cell presence or position within the ovariole. Instead we show that ovariole number evolution has been shaped by a series of transitions between variable and invariant states, with multiple independent lineages evolving to have almost no variation in ovariole number. We highlight the implications of these invariant lineages on our understanding of the specification of ovariole number during development, as well as the importance of considering developmental processes in theories of life-history evolution.
\end{abstract}

\section{Introduction}

Offspring number is a fundamental parameter in the study of life-history ${ }^{1}$. This number differs widely between organisms ${ }^{1}$, and its variation is the foundation for several hypotheses about life-history evolution, including the prediction that there is an evolutionary trade off between the number of offspring and their size (e.g. egg size $)^{1-3}$. In insects, the number of egg-producing compartments in the ovary, called ovarioles, has been used as a proxy for potential offspring number in the study of life-history ${ }^{4-6}$. However, without an understanding of the phylogenetic distribution of ovariole number, this hypothesized relationship cannot be assessed across insects. Here we tested for the presence of a general trade off between ovariole number and egg size by collecting thousands of records of ovariole number from the published literature, placing them in a phylogenetic context, and comparing them to other datasets of insect reproductive morphology. 
The insect female reproductive system includes a pair of ovaries, each of which contains a number of ovarioles ${ }^{7}$ (Fig 1a). Each ovariole consists of an anterior germarium containing the stem cell niche or resting oogonia, developing oocytes arranged in an ontogenic series from anterior to posterior, and a posterior connection to a common oviduct. The number of ovarioles varies across species ${ }^{6}$, and can vary across individuals in a population ${ }^{4}$, as well as between the left and right ovary within a single individual ${ }^{8}$. Therefore total ovariole number may be an even or odd integer for an individual female insect. In addition to variation in the number of ovarioles, the tissue morphology within ovarioles varies across insects, and has been classified into several modes of oogenesis based on the presence and position of special nutritive cells called nurse cells ${ }^{7}$.

Here we compiled 3355 records of ovariole number from across 28 orders, 301 families, and 2103 species of insects. We combined these data with published datasets of egg $\operatorname{size}^{9}$, fecundity ${ }^{10}$, body size ${ }^{11}$, and mode of oogenesis ${ }^{7}$ to test hypotheses about reproductive evolution. In these analyses we used an existing phylogeny of insects ${ }^{12}$ to analyze evolutionary patterns in ovariole number, and found that hypotheses about reproductive trait evolution do not hold generally true across insects. Instead we observe that the phylogenetic distribution of ovariole number suggests a model where the developmental mechanisms that govern ovariole number have shifted between variable and invariant states several times over the course of insect evolution. We propose that the developmental mechanisms used to establish ovariole number in well-studied insects such as Drosophila melanogaster are unlikely to regulate ovariole number in all insects.

\section{Methods}

\section{Gathering trait data}

We searched the published literature for references to insect ovariole number using a predetermined set of 131 search terms, entered into Google Scholar (scholar.google.com) between June and October of 2019. Each search term consisted of an insect taxonomic group and the words "ovariole number". This list was created to include all insect orders, many large insect families, and groups well-represented in the insect egg dataset ${ }^{9}$. For each search term, we evaluated all publications in the first page of results (ten publications). For 61 search terms that had a large number of informative hits, significant representation in the egg dataset, or that corresponded to very speciose groups, we evaluated an additional 20 publications. The list of search terms is available in the supplementary file 'ovariole_number_search_terms.tsv'.

Using this approach, we gathered 3355 records for ovariole number from 448 publications, listed in the supplementary file 'ovariole_number_bibliography.pdf'. We matched these records to additional taxonomic information using the software TaxReformer ${ }^{13}$. For all subsequent analyses, we excluded observations made in non-reproductive individuals from eusocial species (workers), as well as two observations that represented significant outliers and could not be validated using additional sources ${ }^{14,15}$. See supplementary methods section 1 for details.

We combined the data we collected on total ovariole number with existing datasets of egg size and shape ${ }^{9}$, insect lifetime fecundity and dry adult body mass ${ }^{10}$, average adult body length per insect family ${ }^{11}$, several lineage-specific measures of adult body size $^{16-20}$, and the mode of oogenesis ${ }^{7}$. See supplementary methods section 3.1 for details.

All continuous traits (ovariole number, egg volume, lifetime fecundity, and all measures of body size) were $\log _{10}$ transformed for subsequent analyses.

\section{Phylogenetic analyses}

The analyses in this manuscript were performed using the insect phylogeny published in Church et al., $2019^{12}$, unless otherwise specified. Analyses of insect family-level ovariole number, egg size, and body size were performed using the insect phylogeny published in Rainford et. al, $2014^{21}$. Analyses of Drosophilidae ovariole number, egg size, and body size were performed using a phylogeny newly assembled for this study. See supplementary methods section 2 for details. 
To evaluate the robustness of our results to uncertainty in the phylogenetic relationships, all Phylogenetic Generalized Least Squares (PGLS) analyses were performed 1000 times over a posterior distribution of trees, using a Brownian Motion based covariance matrix in the R package ape (version 5.4.1) ${ }^{22}$ and nlme (version 3.1.151) ${ }^{23}$. For regressions at the species and genus level, we reshuffled and matched records for each iteration to account for variation across records for the same taxon. For regressions at the family level, we recalculated the average ovariole number per insect family, randomly downsampling the representation for each family by half. To weight traits by body size, we calculated the phylogenetic residuals ${ }^{24}$ of each trait to body size, and then compared the evolution of these residuals using a PGLS regression. See supplementary methods section 3.2 for details.

For two regressions comparing egg size to ovariole number while accounting for adult body size, we tested alternative hypotheses of evolution by simulating new data. We considered two such hypotheses: no evolutionary correlation with ovariole number, and a strong correlation with ovariole number (slope of -1). For each trait we simulated 1,000 datasets using evolutionary parameters fit under a Brownian Motion model in the R packages geiger (version 2.0.7) ${ }^{24}$, and phylolm (version 2.6.2) ${ }^{25}$.

Ancestral state reconstruction of oogenesis mode was performed with the R package corHMM (version $1.22)^{26}$, and models of trait evolution were compared using the R package Ouwie (version 1.57) ${ }^{27}$. Ancestral state reconstruction and model comparison were repeated 100 times over a posterior distribution of trees and resampling data to account for variation across records for the same taxon. See supplementary methods section 4.3 .

Other comparisons of model fit were performed using the $\mathrm{R}$ package geiger (version 2.0.7) ${ }^{28}$ and validated using a parametric bootstrap with the R package arbutus (version 0.1$)^{29}$. See supplementary methods section 5.1 .

Analyses of evolutionary rate were performed using BAMM (version 2.5.0) ${ }^{30}$. For this analysis, we calculated the average ovariole number ( $\log _{10}$ transformed) for each genus present in the phylogeny (507 taxa). We used the R package BAMMtools (version 2.1.7) ${ }^{31}$ to select appropriate priors, and ran BAMM for the maximum number of generations $\left(2 * 10^{9}\right)$, sampling every $10^{6}$ generations. Convergence was evaluated both visually (Fig. S10) and numerically. Running BAMM for the maximum possible number of generations and selecting the optimum burn-in (Fig. S11) resulted in an effective size for the number of shifts of 482.51, and for loglikelihood of 149.15. Repeated BAMM analyses showed similar distributions of high and low rate regimes, indicating the implications for ovariole number evolution are robust to uncertainty in rate estimates. See supplementary methods section 5.2 for details.

We visualized the results from the BAMM analysis to establish a threshold $\left(10^{-4}\right)$ for assigning a binary rate regime to each node in the phylogeny, categorizing them as above (variable) or below (invariant) a threshold that separates these two peaks.

\section{Data availability}

The dataset of insect ovariole number is available at Dryad, doi:10.5061/dryad.59zw3r253.

\section{Code availability}

The code and phylogenetic trees required to reproduce all the analyses, figures, and generate the manuscript files are provided at 'https://github.com/shchurch/insect_ovariole_number_evolution_2020', commit 9876387. All analyses performed in R (version 4.0.3) were done so in a clean environment, built with conda (version 4.9.2), and instructions for rebuilding this environment are provided in the same repository.

\section{Statistical significance}

All phylogenetic regressions were performed using both the maximum clade credibility (MCC) tree. We considered a relationship significant when the MCC p-value was below the threshold 0.01. To assess the 
robustness of results to uncertainty in phylogenetic relationships, we also report the number of regressions over the posterior distribution of phylogenetic trees that gave a significant result (see Table S1).

For two comparisons, we validated that our tests had sufficient statistical power using the selected threshold by comparing the distribution of p-values from regressions of observed data to regressions of data simulated under alternative hypotheses. We compared the results of analyses of our observed to those based on simulated data to evaluate the likelihood of false positives (comparing to data simulatd under no correlation) and false negatives (comparing to data simulated with strong correlation).

Model comparisons of trait evolution were also performed over a posterior distribution and accounting for phenotypic uncertainty. For these analyses, we considered a model to have significantly better fit the data than other models when the difference in the corrected Akaike Information Criterion (AICc) was greater than two in every analysis iteration.

\section{Results}

\section{Ovariole number diversity}

Ovariole number varies by at least four orders of magnitude across insects (Fig. 1b). We identify seven insect families with species that have been reported to have more than 1,000 total ovarioles, including several eusocial insects (e.g. queens of the termite species Hypotermes obscuriceps, Blattodea: Termitidae ${ }^{32}$, and several ant species, Hymenoptera: Formicidae) ${ }^{33,34}$ and non-eusocial insects (e.g. the blister beetle Meloe proscarabaeus, Coleoptera: Meloidae) ${ }^{35}$. We also find two independent lineages that have evolved to have only one functional ovariole: dung beetles in the tribe Scarabaeinae (Coleoptera: Scarabaeidae) ${ }^{36}$, and grass flies in the genus Pachylophus (Diptera: Chloropidae) ${ }^{37,38}$. In these insects one of the two ovaries presumably established during embryogenesis is reported to atrophy during development ${ }^{38,39}$, resulting in an asymmetric adult reproductive system. 


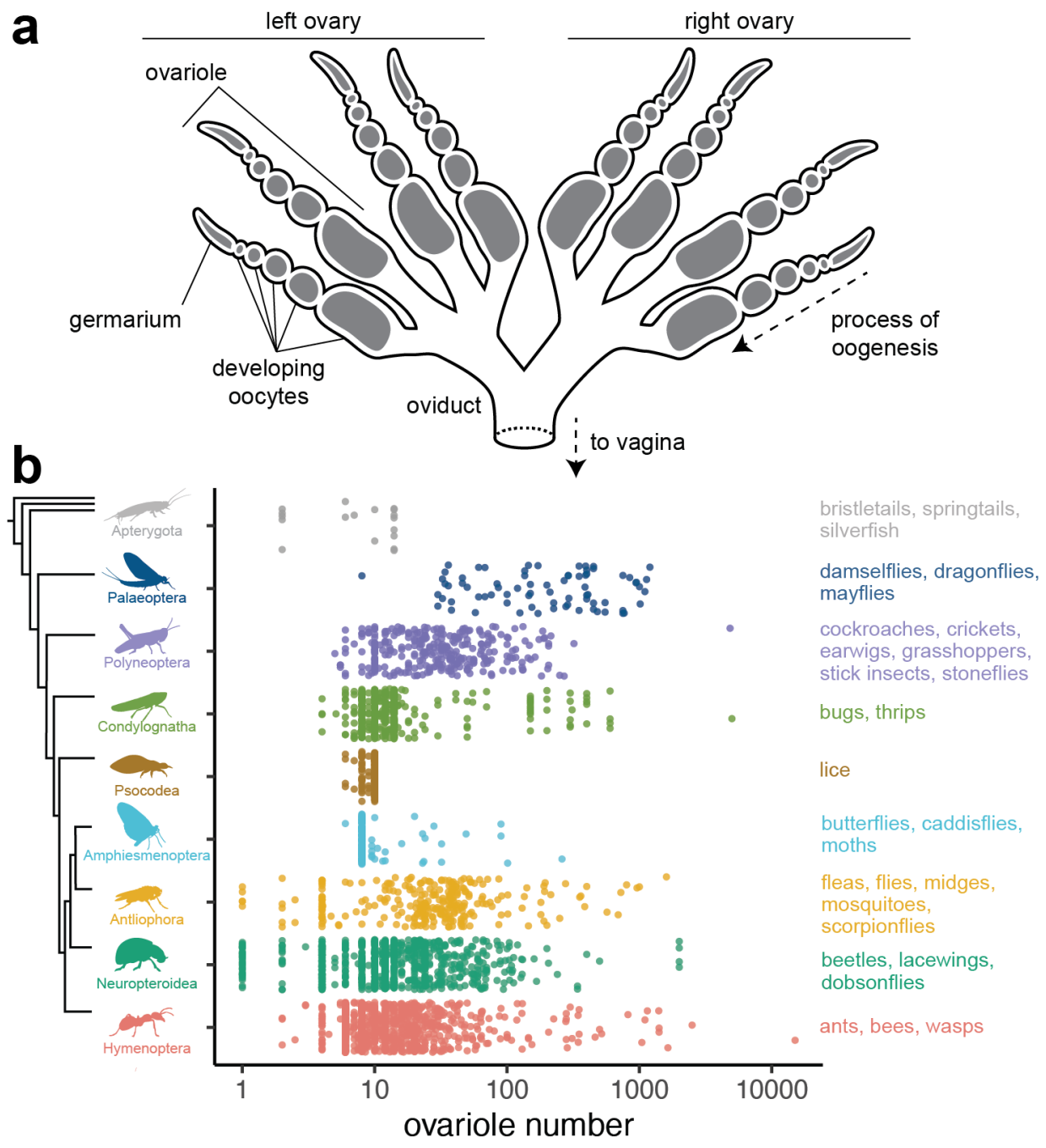

Figure 1: The diversity of ovariole number across insects. a, Schematic of a generalized insect female reproductive system, showing a pair of ovaries, each with four ovarioles. b, The range of total adult ovariole number, $\log _{10}$ scale, across nine groups of insects, arranged with random jitter on the y-axis within each group. Groups are, from top to bottom: Apterygota, Palaeoptera, Polyneoptera, Condylognatha, Psocodea, Amphiesmenoptera, Antliophora, Neuropteroidea, and Hymenoptera.

\section{Ovariole number, egg size, and body size}

Ovariole number has been hypothesized to be negatively correlated with egg size ${ }^{5,19,40}$. This hypothesis is based on the predictions that (1) female reproduction is resource-limited, therefore egg size should trade off with egg number, and (2) ovariole number can serve as a proxy for egg number ${ }^{2,40}$. We did not observe a significant negative relationship when comparing egg size and ovariole number across insect species (Fig. 2a, Table S1, p-value 0.195, $\mathrm{n}=306$ ). We also compared egg size and ovariole number across insect genera and did not observe a significant relationship (Fig. S1, p-value 0.066, $\mathrm{n}=482$ ).

Given that this predicted relationship is often conditioned on taking into account adult body size ${ }^{19,41}$, we combined data on ovariole number and egg size with several measurements of body size, and applied a size correction that accounts for phylogenetic relationships ${ }^{42}$. When accounting for adult body mass, we observed a significant negative relationship between egg size and ovariole number (Fig. 2b, S2, p-value 0.003, n=61). To evaluate the robustness of this result, we repeated the analysis 1000 times, taking into account uncertainty 
in both the phylogeny and trait measurements. Out of 1000 regressions, 995 indicated a significant negative relationship (Table S1). We performed the same comparison accounting for adult body length, and likewise observed a significant negative relationship (Fig. S3, p-value $<0.001, \mathrm{n}=126$ ), supported by 966 of 1000 repeated analyses (Table S1).

We further explored these results using two methods: First, to evaluate our findings against alternative evolutionary hypotheses, we compared these results to regressions based on simulated data. Our results showed that when considering body size, the slope of the regression of egg size and ovariole number is lower than we would expect to observe by chance, as assessed by comparing to data simulated with no evolutionary correlation (Fig. S4). However, for both adult body length and dry mass, the slope of the regressions on observed data are not within the range that would be expected under a strong negative correlation (slope of -1 in log-log space, Fig. S4). This suggests the presence of a weak evolutionary relationship between ovariole number and egg size, when accounting for body size.

Second, we assessed the relationship between egg size and ovariole number, accounting for body size, within four subclades of insects. We found that across Drosophilidae fly species, egg size is indeed strongly negatively correlated with ovariole number when accounting for body size (Fig. 2c, Table S2, p-value $<0.001, \mathrm{n}=30$ ). For grasshoppers and crickets (Orthoptera), beetles (Coleoptera), and wasps (Hymenoptera), we observed no significant relationship between ovariole number and egg size, even when accounting for body size (Fig. 2d, Table S2, S5, Orthoptera: p-value 0.485, $\mathrm{n}=40$, Coleoptera: $\mathrm{p}$-value 0.384, $\mathrm{n}=30$, Hymenoptera: $\mathrm{p}$-value $0.139, \mathrm{n}=21$ ). This indicates that, while a strong negative correlation between egg size and ovariole number exists for some insects, it does not represent a universal pattern across insect clades.

Finally, we tested whether ovariole number is positively correlated with adult body size, and in contrast to previous studies $^{4}$, we found no correlation between ovariole number and adult body mass or length across insects (Fig. S6, Table S3, body mass: p-value $0.618, n=61$, body length: $\mathrm{p}$-value $0.031, \mathrm{n}=98$ ). Of the four subclades considered, only insects in the order Orthoptera had a positive relationship between body size and ovariole number (Table S3, p-value 0.001, $\mathrm{n}=40$ ). 

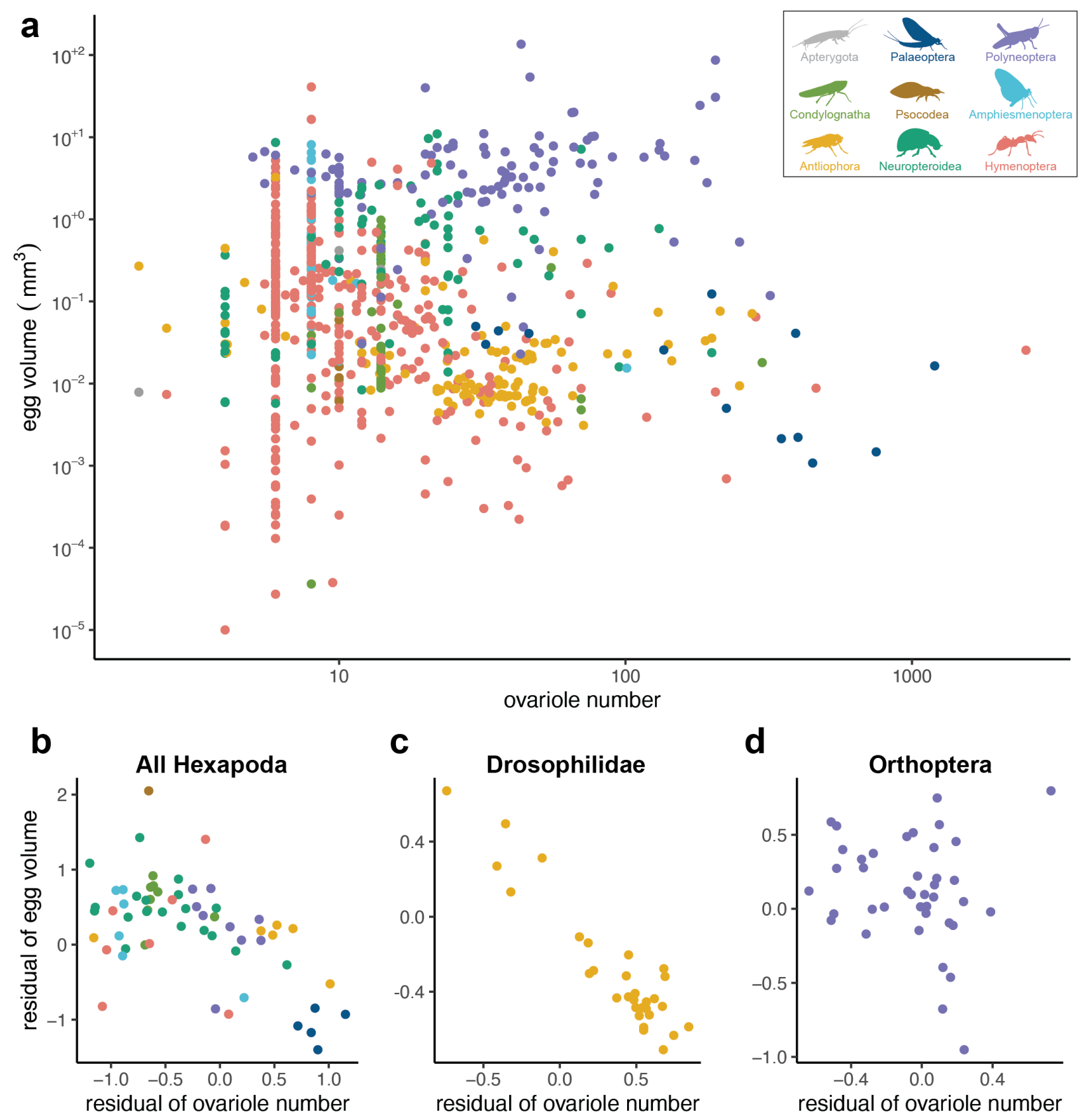

Figure 2: Tests of the hypothesized trade off between egg size and ovariole number. a, Egg volume $\left(\mathrm{mm}^{3}\right)$ and ovariole number, both $\log _{10}$ scale; points represent insect species. See section Modeling ovariole number evolution for discussion of the enrichment of certain low values of ovariole number (points appearing vertically arranged) b, Egg volume and ovariole number, residuals to dry adult body mass, points represent genera. c, Drosophilidae egg volume and ovariole number, residuals to thorax length, points represent species. d, Orthoptera egg volume and ovariole number, residuals to body length, points represent genera. 


\section{Ovariole number and fecundity}

If the hypothesized trade off between the number and size of offspring is true for insects, then one explanation for the lack of a consistent negative relationship between ovariole number and egg size is that ovariole number may not be a reasonable proxy for offspring number. Previous research has shown that, across individuals within the same species, ovariole number is correlated with certain measurements of fecundity, such as maximum daily rate of egg production for Drosophila, ${ }^{43,44}$ but not others, such as lifetime fecundity ${ }^{45}$ or fitness in competition assays ${ }^{46}$. Few studies have compared fecundity and ovariole number across species ${ }^{41}$, likely due to the difficulties of measuring fecundity consistently across insects, many of which lay eggs singly and continuously rather than in distinct clutches.

Using a previously reported dataset of lifetime fecundity measurements across insects ${ }^{10}$, we assessed the relationship between lifetime fecundity and ovariole number. We observed a significant positive relationship (Fig. 3, p-value $0.002, \mathrm{n}=65$ ), however, a substantial fraction of repeat analyses show these results are not robust to uncertainty (733 of 1000 regressions are not significant, Table S4). We note that this relationship is largely defined by the absence of insects with high ovariole number and low fecundity (Fig. 3, empty bottom right corner), while for insects with low ovariole number, fecundity varied over more than three orders of magnitude. We interpret our results, in conjunction with those previously reported, to suggest that ovariole number, when variable across insects in a lineage, may be one factor among many influencing the number of eggs produced. However, we caution against using ovariole number as a direct mathematical proxy for offspring number.

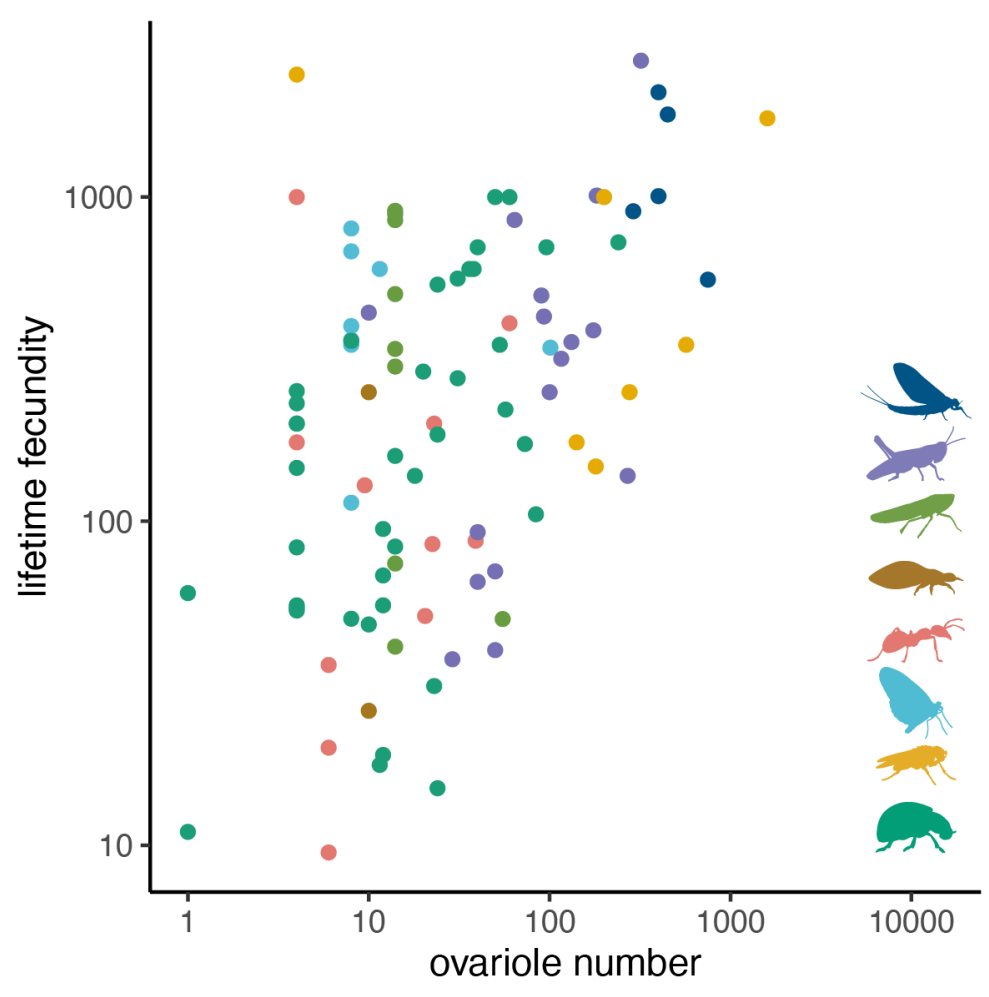

Figure 3: The relationship between lifetime fecundity and ovariole number. Both values are shown on a $\log _{10}$ scale. Points represent insect genera and are colored according to the groups shown in Fig. 1b.

\section{Evolution of nurse cells}

In addition to the number of ovarioles, insect ovary morphology has been classified into several modes of oogenesis based on the presence and position of nutritive cells called nurse cells ${ }^{7}$, (Fig. 4a). Egg formation 
in the well-studied species D. melanogaster is an example of a meroistic oogenesis mode, meaning that its ovarioles contain nurse cells of germ line origin that are connected to developing oocytes via cytoplasmic bridges ${ }^{47}$. In insects with a polytrophic arrangement, these nurse cells are clonally related and immediately adjacent to each oocyte. An alternative arrangement is seen in telotrophic meroistic ovaries, where oocytes in each ovariole are connected to a common pool of nurse cells located in the germarium ${ }^{7}$. Meroistic ovaries are thought to have evolved from an ancestral panoistic mode, meaning they lack nurse cells ${ }^{7}$. Using a previously published set of descriptions of these oogenesis modes across insects ${ }^{7}$, we reconstructed the evolutionary transitions between these states. Consistent with previous analyses ${ }^{7}$, we found that the ancestral insect likely had panoistic ovaries (lacking nurse cells), with several independent shifts to both telotrophic and polytrophic meroistic modes, and at least two reversals from meroistic back to panoistic (Figs. 4b, S8).

Using this ancestral state reconstruction, we then compared models of trait evolution to test whether evolutionary transitions in oogenesis mode helped explain the diversification of ovariole number and egg morphology. We found that, for the traits studied here, models that take into account evolutionary changes in mode of oogenesis do not consistently demonstrate a significant improvement over models that do not take these changes into account $(\Delta \mathrm{AIC}<2$, Table S5). In other words, the evolution of nurse cells and their position within the ovary do not explain the diversification of egg size, egg shape, or ovariole number.

To analyze the robustness of these results to uncertainty in the tree topology and in the inference of ancestral states, we repeated each analysis over a posterior distribution of trees. For egg asymmetry and curvature, but not for volume or aspect ratio, we observed a few iterations where a model that takes into account oogenesis mode evolution was significantly favored over models that did not $(\Delta \mathrm{AIC}>2$, Table S5). However, this result was infrequent over 100 repetitions of the analysis. We therefore interpret these results as suggestive of a possible relationship between mode of oogenesis and egg asymmetry and curvature, but one which cannot be confirmed given the current data available.

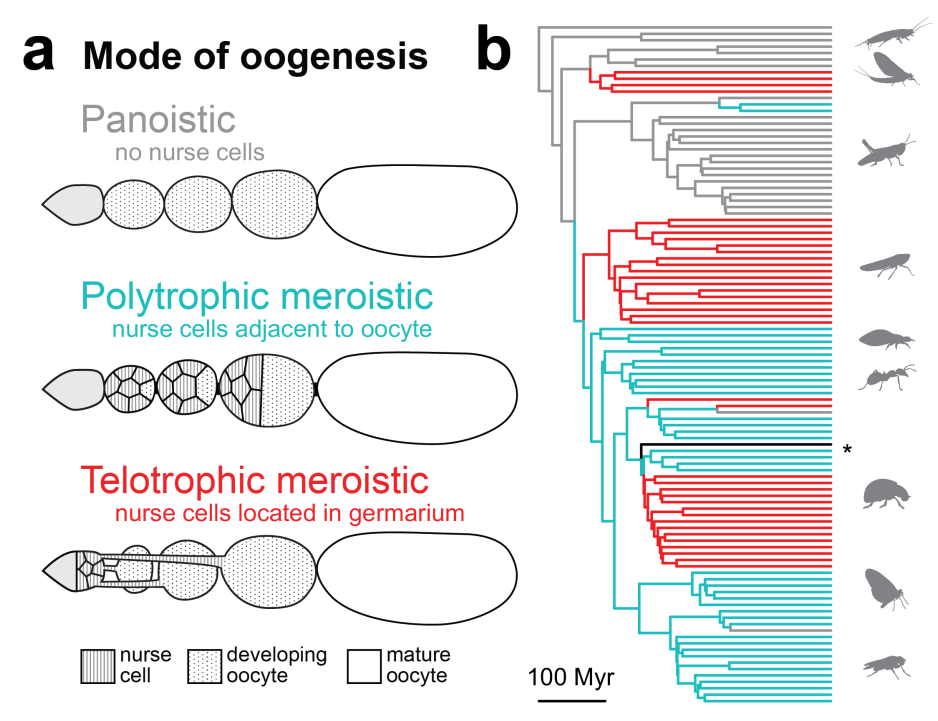

Figure 4: The evolution of the presence and position of nurse cells. a, Insect oogenesis was categorized into several modes by Büning ${ }^{7}$ based on the presence and position of nurse cells. b, Phylogenetic reconstruction of mode of oogenesis. Scale bar indicates 100 million years (Myr). Gray = panoistic ovaries, without nurse cells, cyan = polytrophic meroistic ovaries, with nurse cells adjacent to maturing oocytes, red $=$ telotrophic meroistic ovaries, with nurse cells located in germaria, black = unique meroistic ovary type observed in Strepsiptera. Insect taxonomic groups are, from top to bottom: Apterygota, Palaeoptera, Polyneoptera, Condylognatha, Psocodea, Hymenoptera, Neuropteroidea, Amphiesmenoptera, and Antliophora. 


\section{Modeling ovariole number evolution}

Using the dataset compiled here and a previously published phylogeny of insects (Fig. 5a) ${ }^{12}$, we modeled the rate of evolutionary change in ovariole number (Figs. S9, S10, S11, S12). We observed substantial rate heterogeneity in the evolution of ovariole number (Fig. S12), meaning that for some lineages ovariole number has evolved rapidly where in others, ovariole number has evolved very slowly or not at all. The most striking example of this are the multiple lineages which have independently evolved invariant or nearinvariant ovariole number across taxa (e.g., nearly all Lepidoptera have exactly eight ovarioles, Fig. 5b, cyan), from an ancestral variable state. These invariant lineages were identified by finding regions of the phylogeny that experience extremely low rates of ovariole number diversification (Figs. S12, S13). Using this approach, we found that invariant ovariole numbers have evolved at least nine times independently across insects, with several subsequent reversals from invariant to variable states (Fig. 5a).

We find that the rate of evolutionary change in ovariole number is correlated with the number of ovarioles: lineages with relatively low ovariole number also experience relatively low degrees of ovariole number change (Fig. S9). This is evidenced by the fact that, of the nine invariant lineages, none have greater than seven ovarioles per ovary (Fig. 5c). However we note that not all insects with low ovariole counts are in invariant lineages; many insects with fewer than 14 total ovarioles are in lineages with relatively high rates of intraand interspecific ovariole number variation (Fig. 5)

Additionally, the distribution of ovariole numbers across insects is enriched for even numbers of total ovarioles (Fig. 5c). While many insects show asymmetries in the number of ovarioles between the left and right ovaries, all of the invariant lineages are symmetric (at 4, 6, 8, 10,12, and 14 total ovarioles). Therefore, invariant lineages have near-zero variation when comparing between species, between individuals within a species, and between the left and right ovary within an individual.

Using these results, we propose a multi-rate model, where the rate of ovariole number evolution differs based on the evolution of a discrete trait representing invariant or variable status. We propose that the evolution of this discrete trait is governed by a model where the likelihood of transitions from a variable to an invariant state is negatively correlated with the current number of ovarioles. Here we demonstrate that a multi-rate Brownian motion model far outperforms a single rate model in fitting the data ( $\Delta$ AICc 1770.93). 


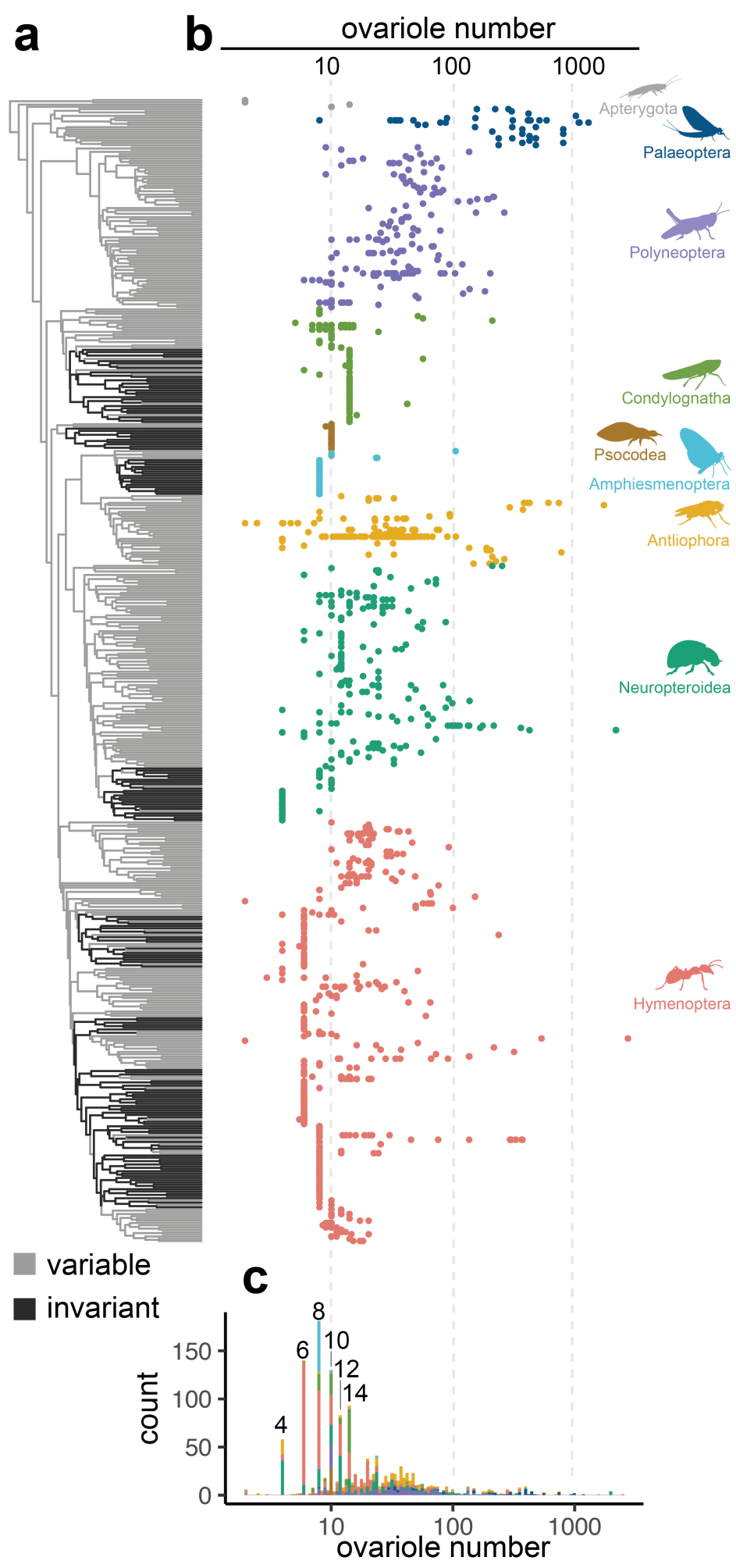

Figure 5: The evolutionary distribution of ovariole number across insects. a, Phylogeny of insect genera, colored according to the inferred rate regime of ovariole number evolution, variable in gray and invariant in black (see Supplementary Methods). b, Total ovariole numbers, shown on a $\log _{10}$ scale and arranged by insect genus according to the phylogeny. Tips with more than one point represent genera with multiple records for total ovariole number in the dataset. c, The distribution of values shown in (b), showing enrichment for even values in the left tail of the distribution. 


\section{Discussion}

A frequently invoked life-history prediction is that, given a finite set of metabolic resources, organisms can either produce few offspring, each with high fitness, or many low-fitness offspring ${ }^{1-3}$. In insects, egg size and ovariole number are often used as proxies for offspring fitness ${ }^{48}$ and number ${ }^{43,44}$, respectively, and therefore it has been predicted that insects with more ovarioles lay smaller eggs than insects with fewer ovarioles ${ }^{5,6,19,40}$. Our results, using a dataset that spans 3355 observations and that takes into account phylogenetic relationships, indicate that a generalized trade-off between insect egg size and ovariole number does not exist (Fig. 2).

Lineages of insects with invariant ovariole number illustrate this point. Despite having the same ovariole number, these lineages contain a range of egg sizes that is comparable to the four orders of magnitude observed across all insects (Fig. 2a). Furthermore, we observed no relationship between the evolutionary rates of change for ovariole number and egg size (Fig. S15). Therefore, if a trade-off between egg size and fecundity exists, factors beyond variation in ovariole number must contribute to fecundity. These factors might include variation in the rate of egg production per ovariole ${ }^{49-52}$, among others ${ }^{53,54}$.

We suggest that considering the evolution of developmental processes that govern ovariole number specification may be more useful in explaining patterns of diversity than predictions based on metabolic trade-offs. The regulation of ovariole number is best understood from research on Drosophila melanogaster, where the number of ovarioles in the adult is determined by cell proliferation and rearrangement during larval development ${ }^{55,56}$. In this species, the number of ovarioles can vary between the left and right ovaries within an individual, as well as across individuals within a population ${ }^{57,58}$. This variation is derived primarily from variation in the number of "terminal filament precursor cells"59,60, as well as from variation in the number of those precursor cells that group together to form the structure that initiates ovariole formation, known as a "terminal filament" 61 . Across species of Drosophila, variation in average adult ovariole number results primarily from variation in the average number of terminal filament precursor cells ${ }^{60}$.

When considering the developmental processes that could give rise to invariant ovariole number, we propose that the major determinants of ovariole number known from Drosophila may not apply. To achieve an invariant ovariole number, these processes might instead include mechanisms for strict counting of individual cells or discrete cell subpopulations. In the former, if the cells that ultimately comprised a terminal filament were derived by mitotic division from a single progenitor, rather than by cellular rearrangements as is the case in Drosophila ${ }^{55}$, then an invariant ovariole number could be achieved via strict control of the number of precursor cells. Alternatively, an invariant ovariole number could be achieved by partitioning the starting population of precursor cells into a tightly regulated number of subpopulations. This would again be a departure from known mechanisms in Drosophila, in which a variable number of precursor cells are gathered into terminal filaments until the population is depleted ${ }^{55,61}$. The determining factor for partitioning the precursor pool could be, for example, a spatially variable morphogen emanating from adjacent tissues ${ }^{62}$ or a reaction-diffusion patterning process ${ }^{63}$ within the developing ovary, as these have been shown to generate fixed numbers of multicellular structures in other developmental contexts ${ }^{64-66}$. These predictions could be tested by characterizing the dynamics of cell number and position across invariant lineages, and making comparisons to corresponding data from their variable relatives.

The evolutionary transitions between variable and invariant ovariole number are reminiscent of other quantitative traits across multicellular life, including patterns of variability and invariance in arthropod segment number ${ }^{67,68}$, vertebrate digit number ${ }^{69,70}$, or the number of angiosperm floral organs ${ }^{71,72}$. Across these systems, the evolutionary history of morphogenetic counting mechanisms is poorly understood. We suggest that insect ovariole number presents an ideal case to study this phenomenon. In particular, we note the evidence that invariance has evolved convergently at least nine times, as well as the evidence of several reversals back to variability from an invariant ancestral state (Fig. 5). These convergent lineages provide an opportunity to test the predictability of evolutionary changes to counting mechanisms, by asking whether convergent evolution of invariance involves convergent canalization of shared molecular mechanisms. 


\section{Acknowledgements}

This work was supported by NSF GRFP DGE1745303 to SHC and funds from Harvard University to support SHC and CGE. NM was supported by the E3 REU program at Harvard University. We thank members of the Extavour Lab for discussion.

\section{Author Contributions}

SHC led the data collection, analysis, and writing of the manuscript. NM performed the initial literature search, data collection, and analysis. BASM assembled all phylogenies for analysis. SD, BASM, and CGE contributed to data analysis, visualization, discussion, and writing.

\section{Competing Interests}

The authors declare no competing interests.

\section{References}

1. Stearns, S. C. The evolution of life histories. (Oxford University Press, 1992).

2. Smith, C. C. \& Fretwell, S. D. The optimal balance between size and number of offspring. The American Naturalist 108, 499-506 (1974).

3. Lack, D. The significance of clutch-size. Ibis 89, 302-352 (1947).

4. Honěk, A. Intraspecific variation in body size and fecundity in insects: A general relationship. Oikos 66, 483-492 (1993).

5. Berrigan, D. The allometry of egg size and number in insects. Oikos 60, 313-321 (1991).

6. Hodin, J. She shapes events as they come: Plasticity in female insect reproduction. in Phenotypic plasticity of insects: Mechanisms and consequences (eds. Whitman, Douglas W \& Ananthakrishnan, T. N.) 423-521 (Science Publishers, 2009).

7. Büning, J. The insect ovary: Ultrastructure, previtellogenic growth and evolution. (Springer Science \& Business Media, 1994).

8. Iwata, K. The comparative anatomy of the ovary in Hymenoptera. Part I. Aculeata. Mushi 29, 1-37 (1955).

9. Church, S. H., Donoughe, S., Medeiros, B. A. de \& Extavour, C. G. A dataset of egg size and shape from more than 6,700 insect species. Scientific Data 6, 1-11 (2019).

10. Gilbert, J. D. J. PhD Thesis: The evolution of parental care in insects. (University of Cambridge, 2007).

11. Rainford, J. L., Hofreiter, M. \& Mayhew, P. J. Phylogenetic analyses suggest that diversification and body size evolution are independent in insects. BMC Evolutionary Biology 16, 8 (2016).

12. Church, S. H., Donoughe, S., Medeiros, B. A. de \& Extavour, C. G. Insect egg size and shape evolve with ecology but not developmental rate. Nature 571, 58-62 (2019).

13. Medeiros, B. A. S. de. TaxReformer. https://github.com/brunoasm/TaxReformer (2019).

14. Su, X. H. et al. Testicular development and modes of apoptosis during spermatogenesis in various castes of the termite Reticulitermes labralis (Isoptera: Rhinotermitidae). Arthropod Structure and Development 44, 630-638 (2015). 
15. Hernandez, L. C., Fajardo, G., Fuentes, L. S. \& Comoglio, L. Biology and reproductive traits of Drymoea veliterna (druce, 1885) (lepidoptera: Geometridae). Journal of Insect Biodiversity 5, 1-9 (2017).

16. Iwata, K. Large-sized eggs in Curculionoidea (Coleoptera). Research Bulletin of Hyogo Agricultural College 7, 43-45 (1966).

17. Iwata, K. \& Sakagami, S. F. Gigantism and dwarfism in bee eggs in relation to the mode of life, with notes on the number of ovarioles. Japanese Journal of Ecology 16, 4-16 (1966).

18. Waloff, N. Number and development of ovarioles of some Acridoidea (Orthoptera) in relation to climate. Physiologia Comparata et Oecologia vol. 3 370-390 (1954).

19. Starmer, W. T. et al. Phylogenetic, geographical, and temporal analysis of female reproductive trade-offs in Drosophilidae. Evolutionary Biology 33, 139-171 (2003).

20. Reinhardt, K., Köhler, G., Maas, S. \& Detzel, P. Low dispersal ability and habitat specificity promote extinctions in rare but not in widespread species: The Orthoptera of Germany. Ecography 28, 593-602 (2005).

21. Rainford, J. L., Hofreiter, M., Nicholson, D. B. \& Mayhew, P. J. Phylogenetic distribution of extant richness suggests metamorphosis is a key innovation driving diversification in insects. PLoS One $\mathbf{9}$, e109085 (2014).

22. Paradis, E., Claude, J. \& Strimmer, K. APE: Analyses of phylogenetics and evolution in R language. Bioinformatics 20, 289-290 (2004).

23. Pinheiro, J., Bates, D., DebRoy, S. \& Sarkar, D. R Core Team (2014) nlme: Linear and nonlinear mixed effects models. R package version 3.1-117. Available at http://cran.r-project.org/package=nlme (2014).

24. Revell, L. J. Phylogenetic signal and linear regression on species data. Methods in Ecology and Evolution 1, 319-329 (2010).

25. Tung Ho, L. S. \& Ané, C. A linear-time algorithm for Gaussian and non-gaussian trait evolution models. Systematic Biology 63, 397-408 (2014).

26. Beaulieu, J. M., O'Meara, B. C. \& Donoghue, M. J. Identifying hidden rate changes in the evolution of a binary morphological character: The evolution of plant habit in campanulid angiosperms. Systematic Biology 62, 725-737 (2013).

27. Beaulieu, J. M., Jhwueng, D.-C., Boettiger, C. \& O'Meara, B. C. Modeling stabilizing selection: Expanding the Ornstein-Uhlenbeck model of adaptive evolution. Evolution 66, 2369-2383 (2012).

28. Harmon, L. J., Weir, J. T., Brock, C. D., Glor, R. E. \& Challenger, W. GEIGER: Investigating evolutionary radiations. Bioinformatics 24, 129-131 (2007).

29. Pennell, M. W., FitzJohn, R. G., Cornwell, W. K. \& Harmon, L. J. Model adequacy and the macroevolution of angiosperm functional traits. The American Naturalist 186, E33-E50 (2015).

30. Rabosky, D. L. Automatic detection of key innovations, rate shifts, and diversity-dependence on phylogenetic trees. PLoS One 9, e89543 (2014).

31. Rabosky, D. L. et al. BAMM tools: An R package for the analysis of evolutionary dynamics on phylogenetic trees. Methods in Ecology and Evolution 5, 701-707 (2014).

32. Bugnion, É. \& Popoff, N. Anatomie de la reine et du roi-termite. Mémoires De La société Zoologique De France 25, 210-232 (1912).

33. Robertson, H. Sperm transfer in the ant Carebara vidua F. Smith (Hymenoptera: Formicidae). Insectes Sociaux 42, 411-418 (1995).

34. Schneirla, T. A comparison of species and genera in the ant subfamily Dorylinae with respect to functional pattern. Insectes Sociaux 4, 259-298 (1957).

35. Büning, J. The trophic tissue of telotrophic ovarioles in polyphage Coleoptera. Zoomorphologie 93, 33-50 (1979). 
36. Richter, P. \& Baker, C. Ovariole number in Scarabaeoidea (Coleoptera: Lucanidae, Passalidae, Scarabaeidae). Proceedings of The Entomological Society of Washington 76, 480-498 (1974).

37. Meier, R., Kotrba, M. \& Ferrar, P. Ovoviviparity and viviparity in the Diptera. Biological Reviews 74, 199-258 (1999).

38. Pollock, J. Viviparous adaptations in the acalyptrate genera Pachylophus (Chloropidae) and Cyrtona (Curtonotidae) (Diptera: Schizophora). Annals of The Natal Museum 37, 183-189 (1996).

39. Pluot, D. Évolution régresive des ovarioles chez les coléoptères Scarabaeinae. Annales de la Société Entomologique de France 15, 575-588 (1979).

40. Montague, J. R., Mangan, R. L. \& Starmer, W. T. Reproductive allocation in the Hawaiian Drosophilidae: Egg size and number. The American Naturalist 118, 865-871 (1981).

41. Stewart, L., Hemptinne, J.-L. \& Dixon, A. Reproductive tactics of ladybird beetles: Relationships between egg size, ovariole number and developmental time. Functional Ecology 5, 380-385 (1991).

42. Revell, L. J. Size-correction and principal components for interspecific comparative studies. Evolution: International Journal of Organic Evolution 63, 3258-3268 (2009).

43. David, J. Nombre d'ovarioles chez Drosophila melanogaster: Relation avec la fecondite et valeur adaptative. Archives De Zoologie Expérimentale et Générale (1970).

44. Boulétreau-Merle, J., Allemand, R., Cohet, Y. \& David, J. Reproductive strategy in Drosophila melanogaster: Significance of a genetic divergence between temperate and tropical populations. Oecologia 53, 323-329 (1982).

45. Schmidt, P. S., Matzkin, L., Ippolito, M. \& Eanes, W. F. Geographic variation in diapause incidence, life-history traits, and climatic adaptation in Drosophila melanogaster. Evolution 59, 1721-1732 (2005).

46. Wayne, M. L., Hackett, J. B. \& Mackay, T. F. Quantitative genetics of ovariole number in Drosophila melanogaster. I. Segregating variation and fitness. Evolution 51, 1156-1163 (1997).

47. King, R. C. Ovarian development in Drosophila melanogaster. (Academic Press, 1970).

48. Koch, L. K. \& Meunier, J. Mother and offspring fitness in an insect with maternal care: Phenotypic trade-offs between egg number, egg mass and egg care. BMC Evolutionary Biology 14, 125 (2014).

49. Drummond-Barbosa, D. \& Spradling, A. C. Stem cells and their progeny respond to nutritional changes during Drosophila oogenesis. Developmental Biology 231, 265-278 (2001).

50. Ables, E. T., Laws, K. M. \& Drummond-Barbosa, D. Control of adult stem cells in vivo by a dynamic physiological environment: Diet-dependent systemic factors in Drosophila and beyond. Wiley Interdisciplinary Reviews: Developmental Biology 1, 657-674 (2012).

51. Mirth, C. K., Alves, A. N. \& Piper, M. D. Turning food into eggs: Insights from nutritional biology and developmental physiology of Drosophila. Current opinion in insect science 31, 49-57 (2019).

52. Wcislo, W. T. The roles of seasonality, host synchrony, and behaviour in the evolutions and distributions of nest parasites in Hymenoptera (Insecta), with special reference to bees (Apoidea). Bioligical Reviews 62, 515-543 (1987).

53. Partridge, L., Fowler, K., Trevitt, S. \& Sharp, W. An examination of the effects of males on the survival and egg-production rates of female Drosophila melanogaster. Journal of Insect Physiology 32, 925-929 (1986).

54. Parker, G. \& Courtney, S. Models of clutch size in insect oviposition. Theoretical Population Biology 26, 27-48 (1984).

55. Godt, D. \& Laski, F. A. Mechanisms of cell rearrangement and cell recruitment in Drosophila ovary morphogenesis and the requirement of bric a brac. Development 121, 173-187 (1995).

56. King, R. C., Aggarwal, S. K. \& Aggarwal, U. The development of the female Drosophila reproductive system. Journal of Morphology 124, 143-165 (1968). 
57. Telonis-Scott, M., McIntyre, L. \& Wayne, M. Genetic architecture of two fitness-related traits in Drosophila melanogaster: Ovariole number and thorax length. Genetica 125, 211-222 (2005).

58. Bergland, A. O., Genissel, A., Nuzhdin, S. V. \& Tatar, M. Quantitative trait loci affecting phenotypic plasticity and the allometric relationship of ovariole number and thorax length in Drosophila melanogaster. Genetics 180, 567-582 (2008).

59. Green II, D. A. \& Extavour, C. G. Convergent evolution of a reproductive trait through distinct developmental mechanisms in Drosophila. Developmental Biology 372, 120-130 (2012).

60. Sarikaya, D. P. et al. Reproductive capacity evolves in response to ecology through common changes in cell number in Hawaiian Drosophila. Current Biology 29, 1877-1884 (2019).

61. Sarikaya, D. P. et al. The roles of cell size and cell number in determining ovariole number in Drosophila. Developmental Biology 363, 279-289 (2012).

62. Lawrence, P. A. \& Struhl, G. Morphogens, compartments, and pattern: Lessons from Drosophila? Cell 85, 951-961 (1996).

63. Kondo, S. \& Miura, T. Reaction-diffusion model as a framework for understanding biological pattern formation. science 329, 1616-1620 (2010).

64. Salazar-Ciudad, I. Tooth morphogenesis in vivo, in vitro, and in silico. Current Topics in Developmental Biology 81, 341-371 (2008).

65. Hatini, V. \& DiNardo, S. Divide and conquer: Pattern formation in drosophila embryonic epidermis. Trends in Genetics 17, 574-579 (2001).

66. Clark, E., Peel, A. D. \& Akam, M. Arthropod segmentation. Development 146, dev170480 (2019).

67. Arthur, W. \& Farrow, M. The pattern of variation in centipede segment number as an example of developmental constraint in evolution. Journal of Theoretical Biology 200, 183-191 (1999).

68. Vedel, V., Chipman, A. D., Akam, M. \& Arthur, W. Temperature-dependent plasticity of segment number in an arthropod species: The centipede Strigamia maritima. Evolution 83 Development 10, 487-492 (2008).

69. Holder, N. Developmental constraints and the evolution of vertebrate digit patterns. Journal of Theoretical Biology 104, 451-471 (1983).

70. Saxena, A., Towers, M. \& Cooper, K. L. The origins, scaling and loss of tetrapod digits. Philosophical Transactions of the Royal Society B: Biological Sciences 372, 20150482 (2017).

71. Ambrose, B. A. \& Purugganan, M. The evolution of plant form. vol. 45 (Wiley, 2012).

72. Kitazawa, M. S. \& Fujimoto, K. A developmental basis for stochasticity in floral organ numbers. Frontiers in Plant Science 5, 545 (2014). 